A-084

J. Chem. Thermodynamics 1978, 10, 959-965

\title{
Heat capacity and thermodynamic properties of diamantane from 5 to $540 \mathrm{~K}$.
}

\author{
EDGAR F. WESTRUM, JR, \\ Department of Chemistry, University of Michigan, Ann Arbor, \\ Michigan 48109, U.S.A. \\ M. ANTHONY McKERVEY, \\ Department of Chemistry, University College, Cork, Eire. \\ JOHN T. S. ANDREWS, R. C. FORT, JR., \\ Liquid Crystal Institute and Department of Chemistry, Kent State \\ University, Kent, Ohio 44240, U.S.A. \\ and \\ TIMOTHY CLARK ${ }^{b}$ \\ Department of Chemistry, The Queens University of Belfast, \\ Northern Ireland BTY SAG, U.K.
}

(Received 1 August 1977; in revised form 10 January 1978)

\begin{abstract}
The heat capacity of diamantane, $\mathrm{C}_{14} \mathrm{H}_{20}$, has been measured by adiabatic calorimetry from 5 to $350 \mathrm{~K}$. The heat capacity is generally sigmate except for a gradual thermal anomaly with a maximum at $35.7 \mathrm{~K}$ and an apparent entropy increment of only $0.8 \mathrm{cal}_{\mathrm{cn}} \mathrm{K}^{-1} \mathrm{~mol}^{-1}$. No explanation of the mechanism of the anomaly is provided. The values of $C_{p}, S^{\circ}$, and $\left\{G^{\circ}(T)-G^{\circ}(0)\right\} / T$ at $298.15 \mathrm{~K}$ are $53.35,47.84$, and $-23.60 \mathrm{cal}_{\mathrm{th}} \mathrm{K}^{-1} \mathrm{~mol}^{-1}$. Thermodynamic properties to $540 \mathrm{~K}$ are included. $\Delta G_{f}^{\circ}(c, 298.15 \mathrm{~K})$ is $26.71 \mathrm{kcal}_{\mathrm{th}} \mathrm{mol}^{-1}$.
\end{abstract}

\section{Introduction}

Diamantane, $\mathrm{C}_{14} \mathrm{H}_{20}$, pentacyclo[7.3.1.14,12.02,7.06,11 $]$ tetradecane (figure 1) as has already been noted is a molecule possessing at least two thermodynamically interesting aspects. $^{(1-4)}$ To enable the provision of complete thermodynamic tables, the heat capacity was studied over the usual cryogenic range.

\section{SAMPLE PROVENANCE}

\section{Experimental}

The preparation and characterization of the sample have been described in the accompanying paper. ${ }^{(1)}$ The sample, transferred from the calorimeter as a liquid in a

a At the University of Michigan, this research was supported in part by the Chemical Thermodynamics Program, Chemistry Section, National Science Foundation under Contract No. GP42525X.

- Present address: Institut Organische Chemie, Erlangen bei Nurnberg, Federal Republic of Germany. 
helium atmosphere after the intermediate-temperature measurements, was subjected to three additional sublimations under vacuum at $310 \mathrm{~K}$ to an ice-cooled receiver.

\section{CALORIMETRIC TECHNIQUE}

The heat capacity of the sample was determined in the Mark II calorimetric cryostat; major features of the cryostat and adjuvant measuring circuitry have been described elsewhere. ${ }^{(5)}$ The sample was contained in a gold-plated copper calorimeter (laboratory designation W-52) with a capsule-type platinum resistance thermometer (A-5) calibrated by the National Bureau of Standards against IPTS-48. An entrant axially symmetrical well contained the removable bifilarly wound heater assembly surrounding the thermometer. The calorimeter had an internal volume of $59.11 \mathrm{~cm}^{3}$, a mass $13.1 \mathrm{~g}$, excluding the thermometer-heater assembly, and was provided with a knifeedged gold-gasketed screw closure fitting previously described. ${ }^{(5)}$ The heat capacity of the empty calorimeter was determined in a separate series of measurements. The heat capacity of the sample was 90 per cent of the total measured heat capacity below $20 \mathrm{~K}$. It decreased to 60 per cent at $140 \mathrm{~K}$ and rose to 80 per cent at $350 \mathrm{~K}$. Buoyancy correction was made on the basis of a crystallographic density of $1.21 \mathrm{~g} \mathrm{~cm}^{-3}$ for $\mathrm{C}_{14} \mathrm{H}_{20}{ }^{(6)}$ Adjustments were made for small differences in gold, copper, Apiezon- $\mathrm{T}$ grease, and helium between measurements on the full and empty calorimeter and contributed less than 0.02 per cent uncertainty even in the $10 \mathrm{~K}$ region. To facilitate thermal equilibration, $44.0 \mathrm{kPa}$ of helium gas was added to the evacuated calorimeter prior to sealing of the sample space in the calorimeter.

\section{Results and discussion}

\section{HEAT CAPACITIES}

The experimental molar heat capacities are compiled in table 1 in chronological sequence so that the approximate temperature increments may usually be deduced from differences between adjacent (mean) temperatures. The results are stated in terms of the IUPAC-1973 molar mass of diamantane as $188.314 \mathrm{~g} \mathrm{~mol}^{-1}$. Adjustments for the finite temperature increments used in the measurements ("curvature corrections") were applied to all values in table 1 by the first term in the series derived by Justice $e t a l .{ }^{(5)}$ The estimated standard deviation of the heat capacities is 8 per cent at $5 \mathrm{~K}, 1$ per cent at $15 \mathrm{~K}$, and 0.07 per cent above $25 \mathrm{~K}$. The curve is sigmate with only a small anomaly near $35.7 \mathrm{~K}$.

\section{THE 35.7 K THERMAL ANOMALY}

As may be seen in figure 1, a gradually increasing excess heat capacity with a maximum at $35.7 \mathrm{~K}$ is observed. The associated enthalpy and entropy increments are summarized in table 2 assuming that the lattice heat capacity-interpolated with a Debye function-shown in figure 2 obtains. These transitional increments seem too small to be accounted for on the basis of molecular disorder of individual molecules although concerted motions are not excluded. The transition is reminiscent-though about ten times smaller-of that observed in paracyclophane and there tentatively attributed to a weakly cooperative disordering phenomenon. ${ }^{(7)}$ 
TABLE 1. Experimental values for the heat capacity of diamantane.

$\left(\mathrm{cal}_{\mathrm{th}}=4.184 \mathrm{~J}\right)$

\begin{tabular}{|c|c|c|c|c|c|c|c|}
\hline$\frac{T}{\mathbf{K}}$ & $\frac{C_{p}}{\mathrm{~K}^{-1} \mathrm{~mol}^{-1}}$ & $\frac{T}{\mathbf{K}}$ & $\frac{C_{p}}{\mathrm{~K}^{-1} \mathrm{~mol}^{-1}}$ & $\frac{T}{\mathrm{~K}} \mathrm{c}$ & $\frac{C_{p}}{c a l_{t b}} \mathrm{~K}^{-1} \mathrm{~mol}^{-1}$ & $\frac{T}{\mathrm{~K}}$ & $\frac{C_{p}}{\mathrm{cal}_{\mathrm{th}} \mathrm{K}^{-1} \mathrm{~mol}^{-1}}$ \\
\hline & & \multicolumn{4}{|c|}{ Low-temperature adiabatic cryostat (Ann Arbor) } & & \\
\hline \multicolumn{2}{|c|}{ Series I } & 223.65 & 36.03 & 6.32 & 0.209 & \multicolumn{2}{|c|}{ Series VI } \\
\hline 49.84 & 9.577 & 233.57 & 38.15 & 7.10 & 0.290 & 32.51 & 7.464 \\
\hline 52.67 & 9.851 & 243.54 & 40.35 & 8.03 & 0.434 & 33.45 & 7.749 \\
\hline 58.03 & 10.380 & & & 9.18 & 0.675 & 34.47 & 7.952 \\
\hline 63.88 & 10.972 & \multicolumn{2}{|c|}{ Series III } & 10.30 & 0.963 & 35.51 & 8.091 \\
\hline 70.37 & 11.590 & 245.46 & 40.79 & 11.46 & 1.203 & 36.56 & 7.982 \\
\hline 77.57 & 12.352 & 257.24 & 43.50 & 12.80 & 1.563 & 37.57 & 8.074 \\
\hline 85.47 & 13.277 & 267.03 & 45.82 & 14.22 & 1.991 & 38.55 & 8.174 \\
\hline 93.97 & 14.283 & 277.11 & 48.21 & 15.77 & 2.506 & & \\
\hline 103.20 & 15.44 & 287.23 & 50.69 & 17.45 & 3.056 & \multicolumn{2}{|c|}{ Series VII } \\
\hline 112.97 & 16.78 & 297.47 & 53.27 & 19.29 & 3.667 & 30.27 & 6.935 \\
\hline 122.81 & 18.19 & 307.73 & 55.87 & 21.29 & 4.330 & 33.61 & 7.777 \\
\hline \multirow[t]{2}{*}{132.61} & 19.66 & 318.03 & 58.52 & 23.59 & 5.058 & 35.17 & 8.070 \\
\hline & & 328.17 & 61.13 & 26.09 & 5.810 & 35.46 & 8.130 \\
\hline \multicolumn{2}{|c|}{ Series II } & 338.17 & 63.76 & 28.82 & 6.551 & 35.69 & 8.039 \\
\hline 132.80 & 19.68 & 347.09 & 66.20 & 31.76 & 7.323 & 35.92 & 7.970 \\
\hline 143.11 & 21.31 & \multirow{2}{*}{\multicolumn{2}{|c|}{ Series IV }} & 35.09 & 7.965 & 36.10 & 7.959 \\
\hline 153.77 & 23.00 & & & 39.10 & 8.285 & 36.24 & 7.962 \\
\hline 164.25 & 24.75 & 131.36 & 19.47 & 42.56 & 8.706 & 36.38 & 7.959 \\
\hline 174.44 & 26.51 & 143.26 & 21.31 & 46.42 & 9.180 & 36.59 & 8.007 \\
\hline 184.41 & 28.30 & & & 51.28 & 9.713 & 36.86 & 8.024 \\
\hline 194.20 & 30.11 & \multicolumn{2}{|c|}{ Series V } & 55.77 & 10.140 & & \\
\hline 203.95 & 32.00 & 4.82 & 0.109 & & & & \\
\hline 213.78 & 33.97 & 5.41 & 0.119 & & & & \\
\hline
\end{tabular}

TABLE 2. Enthalpy in the transition region of diamantane $\left(\mathrm{cal}_{\mathrm{th}}=4.184 \mathrm{~J}\right)$

\begin{tabular}{|c|c|c|c|c|}
\hline $\begin{array}{l}\text { Designation } \\
\text { (number of detn's) }\end{array}$ & $\frac{T_{1}}{\mathrm{~K}}$ & $\frac{T_{f}}{\mathbf{K}}$ & $\frac{H^{\circ}\left(T_{f}\right)-H^{\circ}\left(T_{1}\right)}{\mathrm{cal}_{\mathrm{tg}} \mathrm{mol}^{-1}}$ & $\frac{H^{\circ}(40 \mathrm{~K})-H^{\circ}(24 \mathrm{~K})}{\mathrm{cal}_{\mathrm{th}} \mathrm{mol}^{-1}}$ \\
\hline \multirow[t]{2}{*}{$\begin{array}{l}\text { Series V (5) } \\
\text { Series VII }(7) \\
\text { Series VI }(7)\end{array}$} & $\begin{array}{l}24.83 \\
28.32 \\
32.72\end{array}$ & $\begin{array}{l}41.25 \\
39.04 \\
36.99\end{array}$ & $\begin{array}{r}101.08 \\
82.68 \\
30.02\end{array}$ & $\begin{array}{l}114.50 \\
114.44 \\
114.56\end{array}$ \\
\hline & & Lattice entha & $\begin{array}{r}\text { Mean val } \\
(40 \mathrm{~K})-H_{\mathrm{L}}^{\circ}(24 \mathrm{~K}\end{array}$ & $\begin{array}{l}114.50 \pm 0.04 \\
112.0\end{array}$ \\
\hline \multicolumn{2}{|c|}{$\Delta S_{\mathrm{t}}^{\circ} / \mathrm{cal}_{\mathrm{th}} \mathrm{K}^{-1} \mathrm{~mol}^{-1}=0.08$} & & & 2.5 \\
\hline
\end{tabular}

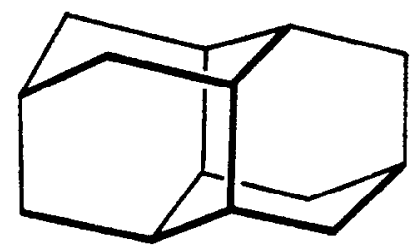

FIGURE 1. Diamantane molecule. 


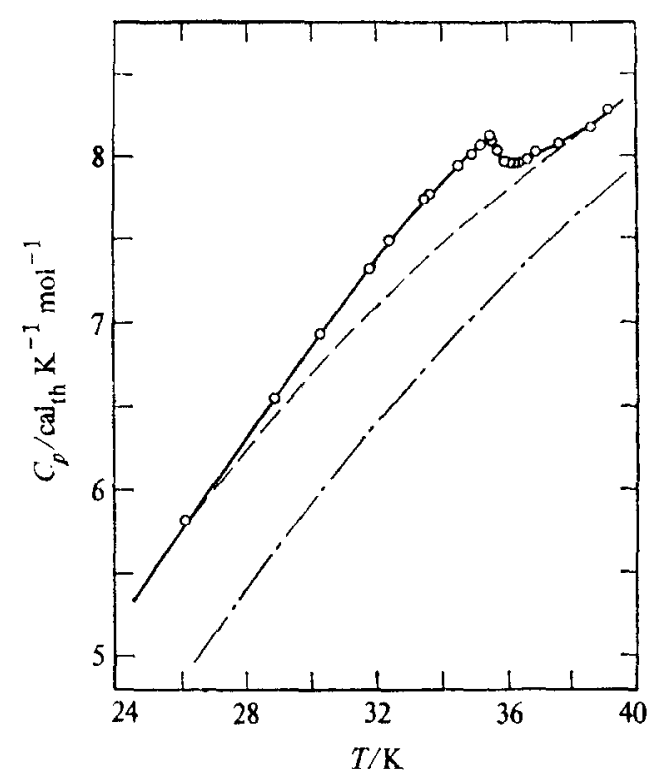

FIGURE 2. $O$, Experimental heat capacities in the region of the thermal anomaly. - - Lattice heat capacity interpolated from Debye characteristic temperature. _..... Heat capacity of adamantane included for comparison.

While handling the sample in glass we noted that fine crystals were unusually susceptible to the attraction of electrostatic fields (such as that generated by rubbing the outside of the glass).

\section{THERMAL FUNCTIONS}

The experimental heat capacities below $410 \mathrm{~K}$ from this paper were combined with those of the related endeavor, ${ }^{(1)}$ and fitted to two overlapping polynomials in temperature by least squares. Values of the thermal functions given in table 3 were obtained by appropriate integrations over the polynomials. Below $5 \mathrm{~K}$, the heat capacity was linearly extrapolated to $T=0$ on a $C_{p} / T$ against $T^{2}$ plot. Appropriate integrations over the several transitions were incorporated into the table. No adjustment was made in the extrapolation process for contributions due to nuclear spin or isotopic mixing; hence, the values are practicable thermal functions for use in ordinary thermochemical calculations. Standard deviations of heat-capacity values selected from the smoothed curve are 0.4 per cent from 10 to $30 \mathrm{~K}$ and 0.08 per cent above this temperature; that of the thermal functions is about 0.08 per cent above $100 \mathrm{~K}$.

\section{THERMOCHEMICAL PROPERTIES}

The standard entropy of formation at $298.15 \mathrm{~K}$ can be calculated from the results of this research together with $S^{\circ}(\mathrm{C}, \mathrm{c}, 298.15 \mathrm{~K})=(1.372 \pm 0.029) \mathrm{cal}_{\mathrm{th}} \mathrm{K}^{-1} \mathrm{~mol}^{-1}$, (B) 
TABLE 3. Thermodynamic functions of diamantane $\left(\mathrm{cal}_{\mathrm{th}}=4.184 \mathrm{~J}\right)$

\begin{tabular}{|c|c|c|c|c|}
\hline$\frac{T}{\mathrm{~K}}$ & $\frac{C_{\mathrm{p}}}{\mathrm{Cal}_{\mathrm{th}}}-\frac{\mathrm{K}^{-1} \mathrm{~mol}^{-1}}{-1}$ & $\frac{S^{\circ}(T)-S^{\circ}(0)}{\mathrm{cal}_{\mathrm{th}} \mathrm{K}^{-1} \mathrm{~mol}^{-1}}$ & $\frac{H^{\circ}(T)-H^{\circ}(0)}{\text { cal }_{\mathrm{th}} \mathrm{mol}^{-1}}$ & $\frac{-\left\{G^{\circ}(T)-H^{\circ}(0)\right\} / T}{\mathrm{cal}_{\mathrm{th}} \mathrm{K}^{-1} \mathrm{~mol}^{-1}}$ \\
\hline \multicolumn{5}{|c|}{ Crystal III } \\
\hline 5 & 0.098 & 0.036 & 0.122 & 0.012 \\
\hline 10 & 0.835 & 0.280 & 2.088 & 0.071 \\
\hline 15 & 2.247 & 0.870 & 9.603 & 0.230 \\
\hline 20 & 3.909 & 1.742 & 24.965 & 0.494 \\
\hline 25 & 5.482 & 2.788 & 48.528 & 0.847 \\
\hline 30 & 6.886 & 3.913 & 79.494 & 1.263 \\
\hline 35 & 8.044 & 5.067 & 117.014 & 1.724 \\
\hline 40 & 8.377 & 6.154 & 157.50 & 2.212 \\
\hline 45 & 9.004 & 7.178 & 201.18 & 2.707 \\
\hline 50 & 9.570 & 8.156 & 247.64 & 3.203 \\
\hline 60 & 10.580 & 9.992 & 348.51 & 4.184 \\
\hline 70 & 11.539 & 11.695 & 459.08 & 5.137 \\
\hline 80 & 12.624 & 13.306 & 579.8 & 6.058 \\
\hline 90 & 13.806 & 14.860 & 711.9 & 6.950 \\
\hline 100 & 15.07 & 16.38 & 856.2 & 7.817 \\
\hline 110 & 16.40 & 17.88 & 1013.5 & 8.664 \\
\hline 120 & 17.80 & 19.36 & 1184.5 & 9.494 \\
\hline 130 & 19.26 & 20.85 & 1369.7 & 10.310 \\
\hline 140 & 20.78 & 22.33 & 1569.9 & 11.115 \\
\hline 150 & 22.37 & 23.82 & 1785.6 & 11.913 \\
\hline 160 & 24.01 & 25.31 & 2017.5 & 12.703 \\
\hline 170 & 25.72 & 26.82 & 2266.1 & 13.489 \\
\hline 180 & 27.49 & 28.34 & 2532.0 & 14.272 \\
\hline 190 & 29.33 & 29.87 & 2816.1 & 15.05 \\
\hline 200 & 31.24 & 31.43 & 3118.9 & 15.83 \\
\hline 210 & 33.22 & 33.00 & 3441.2 & 16.61 \\
\hline 220 & 35.27 & 34.59 & 3783.6 & 17.39 \\
\hline 230 & 37.39 & 36.21 & 3135.8 & 18.18 \\
\hline 240 & 39.58 & 37.84 & 4531.6 & 18.96 \\
\hline 250 & 41.83 & 39.50 & 4938.6 & 19.75 \\
\hline 260 & 44.14 & 41.19 & 5367.9 & 20.54 \\
\hline 270 & 46.49 & 42.90 & 5821.0 & 21.34 \\
\hline 280 & 48.89 & 44.63 & 6297.9 & 22.14 \\
\hline 290 & 51.33 & 46.39 & 6799.0 & 22.94 \\
\hline 300 & 53.81 & 48.17 & 7324.7 & 23.75 \\
\hline 273.15 & 47.24 & 43.44 & 5821.04 & 21.34 \\
\hline 298.15 & 53.35 & 47.84 & 7226.53 & 23.60 \\
\hline 310 & 56.34 & 49.98 & 7875.4 & 24.57 \\
\hline 320 & 58.90 & 51.80 & 8451.6 & 25.39 \\
\hline 330 & 61.49 & 53.66 & 9053.5 & 26.22 \\
\hline 340 & 64.10 & 55.53 & 9681.4 & 27.06 \\
\hline 350 & 66.73 & 57.43 & 10335.6 & 27.90 \\
\hline
\end{tabular}


TABLE 3-continued

\begin{tabular}{|c|c|c|c|c|}
\hline$\frac{T}{\mathbf{K}}$ & $\frac{C_{p}}{\mathrm{cal}_{\mathrm{th}} \mathrm{K}^{-1} \mathrm{~mol}^{-1}}$ & $\frac{S^{\circ}(T)-S^{\circ}(0)}{\mathrm{Cal}_{\mathrm{th}} \mathrm{K}^{-1} \mathrm{~mol}^{-1}}$ & $\frac{H^{\circ}(T)-H^{\circ}(0)}{\mathrm{cal}_{\mathrm{th}} \mathrm{mol}^{-1}}$ & $\frac{-\left\{G^{\circ}(T)-H^{\circ}(0)\right\} / T}{\mathrm{cal}_{\mathrm{th}} \mathrm{K}^{-1} \mathrm{~mol}^{-1}}$ \\
\hline \multicolumn{5}{|c|}{ Crystal III-continued } \\
\hline $\begin{array}{l}360 \\
370 \\
380 \\
390 \\
400\end{array}$ & $\begin{array}{l}69.38 \\
72.06 \\
74.79 \\
77.61 \\
80.54\end{array}$ & $\begin{array}{l}59.34 \\
61.28 \\
63.24 \\
65.22 \\
67.22\end{array}$ & $\begin{array}{l}11016.2 \\
11723.3 \\
12457.5 \\
13219.5 \\
14010.1\end{array}$ & $\begin{array}{l}28.74 \\
29.60 \\
30.46 \\
31.32 \\
32.19\end{array}$ \\
\hline $407.22^{a}$ & $(82.4090)^{\circ}$ & 68.66 & 14598.3 & 32.81 \\
\hline \multicolumn{5}{|c|}{ Crystal II ${ }^{\circ}$} \\
\hline $\begin{array}{l}407.22^{\circ} \\
420.00 \\
440.43^{\circ}\end{array}$ & $\begin{array}{l}(82.94)^{\circ} \\
85.55 \\
(89.79)^{\circ}\end{array}$ & $\begin{array}{l}71.25 \\
73.87 \\
78.04\end{array}$ & $\begin{array}{l}15653.5 \\
16737 \\
18529\end{array}$ & $\begin{array}{l}32.81 \\
34.02 \\
35.97\end{array}$ \\
\hline \multicolumn{5}{|c|}{ Crystal I d } \\
\hline $\begin{array}{l}440.43^{a} \\
450 \\
460 \\
470 \\
480\end{array}$ & $\begin{array}{l}92.26^{\circ} \\
93.81 \\
95.33 \\
96.87 \\
98.43\end{array}$ & $\begin{array}{l}82.90 \\
84.90 \\
86.98 \\
89.04 \\
91.10\end{array}$ & $\begin{array}{l}20669 \\
21560 \\
22506 \\
23467 \\
24444\end{array}$ & $\begin{array}{l}35.97 \\
36.99 \\
38.05 \\
39.11 \\
40.18\end{array}$ \\
\hline $\begin{array}{l}490 \\
500 \\
517.92^{a}\end{array}$ & $\begin{array}{c}99.99 \\
101.56 \\
(103.85)^{\circ}\end{array}$ & $\begin{array}{l}93.14 \\
95.18 \\
98.80\end{array}$ & $\begin{array}{l}25436 \\
26444 \\
28284\end{array}$ & $\begin{array}{l}41.24 \\
42.29 \\
44.19\end{array}$ \\
\hline \multicolumn{5}{|c|}{ Liquid • } \\
\hline $\begin{array}{l}517.922^{\circ} \\
520 \\
530 \\
540\end{array}$ & $\begin{array}{l}(104.79)^{b} \\
105.17 \\
106.98 \\
108.61\end{array}$ & $\begin{array}{l}102.81 \\
103.23 \\
105.25 \\
107.26\end{array}$ & $\begin{array}{l}30360 \\
30569 \\
31630 \\
32708\end{array}$ & $\begin{array}{l}44.19 \\
44.44 \\
45.57 \\
46.69\end{array}$ \\
\hline
\end{tabular}

Assuming transition to be truly isothermal.

- Slightly extrapolated to $T_{t}$.

- Maximum $C_{p}>7 \times 10^{2} \mathrm{cal}_{\mathrm{th}} \mathrm{K}^{-1} \mathrm{~mol}^{-1} ; \Delta S_{\mathrm{t}}^{\circ}(\mathrm{III}=\mathrm{II})=\left(10.8_{\mathrm{t}} \pm 0.06\right) \mathrm{cal}_{\mathrm{th}} \mathrm{K}^{-1} \mathrm{~mol}^{-1}$.

${ }^{d}$ Maximum $C_{y}>5 \times 10^{3} \mathrm{cal}_{\mathrm{th}} \mathrm{K}^{-1} \mathrm{~mol}^{-1} ; \Delta S_{\mathrm{t}}^{0}(\mathrm{II}=\mathrm{I})=\left(20.3_{5} \pm 0.3\right) \mathrm{cal}_{\mathrm{th}} \mathrm{K}^{-1} \mathrm{~mol}^{-1}$.

- Maximum $C_{\mathrm{p}}>5 \times 10^{3} \mathrm{cal}_{\mathrm{th}} \mathrm{K}^{-1} \mathrm{~mol}^{-1} ; \Delta S(\mathrm{I}=1)=\left(16.7_{\mathrm{a}} \pm 0.15\right) \mathrm{cal}_{\mathrm{th}} \mathrm{K}^{-1} \mathrm{~mol}^{-1}$. (as elsewhere in this paper, the uncertainty indices are standard deviations).

and $S^{\circ}\left(\mathrm{H}_{2}, \mathrm{~g}, 298.15 \mathrm{~K}\right)=(31.207 \pm 0.008) \mathrm{cal}_{\mathrm{th}} \mathrm{K}^{-1} \mathrm{~mol}^{-1}{ }^{(8)} \uparrow$ The result is

$$
\Delta S_{\mathrm{f}}^{\circ}\left(\mathrm{C}_{14} \mathrm{H}_{20}, \mathrm{c}, 298.15 \mathrm{~K}\right)=(-283.44 \pm 0.42) \mathrm{cal}_{\mathrm{th}} \mathrm{K}^{-1} \mathrm{~mol}^{-1} \text {. }
$$

The enthalpy of formation has been determined by oxygen bomb calorimetry in several laboratories. ${ }^{(4,9,10)}$ The $\Delta H_{\mathrm{f}}^{\circ}\left(\mathrm{C}_{14} \mathrm{H}_{20}, \mathrm{c}, 298.15 \mathrm{~K}\right)$ value of Carson et al. ${ }^{(9)}$ is $(-67.55 \pm 0.42) \mathrm{kcal}_{\mathrm{th}} \mathrm{mol}^{-1}$, that of Clark et al..$^{(4)}$ is $\left(-55.5_{3} \pm 0.58\right) \mathrm{kcal}_{\mathrm{th}}$ $\mathrm{mol}^{-1}$, and that of Clark and McKervey ${ }^{(10)}$ is $(-57.80 \pm 0.61) \mathrm{kcal}_{\mathrm{th}} \mathrm{mol}^{-1}$. We select the value of Clark and McKervey as the most reliable. Hence,

$$
\Delta H_{\mathrm{f}}^{\circ}\left(\mathrm{C}_{14} \mathrm{H}_{20}, \mathrm{c}, 298.15 \mathrm{~K}\right)=(-57.80 \pm 0.61) \mathrm{kcal}_{\mathrm{th}} \mathrm{mol}^{-1} \text {. }
$$

$\dagger$ Throughout this paper $\mathrm{cal}_{\mathrm{th}}=4.184 \mathrm{~J}$. 
This value may be combined with the $\Delta S_{\mathrm{f}}^{\circ}$ to yield the standard Gibbs energy of formation:

$$
\Delta G_{\mathrm{f}}^{\mathrm{o}}\left(\mathrm{C}_{14} \mathrm{H}_{20}, \mathrm{c}, 298.15 \mathrm{~K}\right)=(26.71 \pm 0.63) \mathrm{kcal}_{\mathrm{th}} \mathrm{mol}^{-1} .
$$

For the gaseous phase, the chemistry of formation is somewhat less secure and the data on which published values are based cannot yet be critically evaluated.

\section{OBSERVATIONS}

Although marked anisotropy in the molecular motions in diamantane below $300 \mathrm{~K}$ has been noted by neutron-diffraction techniques, ${ }^{(1)}$ neither this nor the potentialenergy calculations ${ }^{(12)}$ shed explanatory insights on the phenomena observed in this study.

In a communication received subsequent to the submission of this paper Jenkins et $a l .{ }^{(13)}$ report on the basis of Raman spectroscopy that a soft librational mode goes to zero at $35 \mathrm{~K}$ and interpret this in terms of collective motions of the diamantane molecules about the principal symmetry axis.

We thank Albert Highe for help with measurements and calculations on the diamantane sample.

\section{REFERENCES}

1. Spinella, G. M.; Andrews, J. T. S.; Bacon, W. E., Jr.; Fort, R. C., Jr.; Sabo, J. J. Chem. Thermodynamics in the press.

2. Clark, T.; Johnston, D. E.; Mackle, H.; McKervey, M. A.; Rooney, J. J. J. Chem. Soc. Chem. Comm. 1972, 1042; and Clark, T.; McKervey, M. A.; Mackle, H.; Rooney, J. J. J. Chem. Soc. Faraday Trans. I 1974, 70, 1279.

3. Schweizer, E. E.; Kim, C. S.; Labaw, C. S.; Murray, W. P. J. Chem. Soc. Chem. Comm. 1973, 7.

4. Clark, T.; Knox, T. M.; Mackle, H.; McKervey, M. A.; Rooney, J. J. J. Am. Chem. Soc. 1975, 97, 3835.

5. Westrum, E. F., Jr.; Furukawa, G. T.; McCullough, J. P. In Experimental Thermodynamics, Vol. 1. McCullough, J. P.; Scott, D. W.: editors. Butterworths: London 1968, p. 133.

6. Karle, I. L.; Karle, J. J. Am. Chem. Soc. 1965, 87, 918.

7. Andrews, J. T. S.; Westrum, E. F., Jr. J. Phys. Chem. 1970, 74, 2170

8. CODATA Key Values for Thermodynamics. CODATA Bulletin No. 17, CODATA, Paris, France, January 1976.

9. Carson, A. S.; Laye, P. G.; Steele, W. V.; Johnston, D. E.; McKervey, M. A. J. Chem. Thermodynamics 1971, 3, 915.

10. Clark, T.; McKervey, M. A. Personal communication (April 1977).

11. Evans, E. H. M.; Hine, R.; Richards, J. P. G. J. Phys. C, Solid State Phys. 1976, 9, 369.

12. Fyfe, C. A.; Harold-Smith, D. J. Chem. Soc. Faraday Trans. II 1975, 71, 967.

13. Jenkins, T. E.; Bates, A. R.; Evans, E. M. H. J. Phys. C, Solid State Phys. 1978, 11, 83. 